Utah State University

DigitalCommons@USU

6-25-2014

\title{
Defects Density of States Model of Cathodoluminescent Intensity and Spectra of Disordered SiO2
}

Amberly Evans Jensen

Utah State University

JR Dennison

Utah State Univesity

Follow this and additional works at: https://digitalcommons.usu.edu/mp_conf

Part of the Condensed Matter Physics Commons

\section{Recommended Citation}

Amberly Evans Jensen and JR Dennison, "Defects Density of States Model of Cathodoluminescent Intensity and Spectra of Disordered SiO2," Abstract 144, Proceedings of the 13th Spacecraft Charging Technology Conference, (Pasadena, CA, June 25-29, 2014), 7 pp.

This Conference Paper is brought to you for free and open access by the Materials Physics at DigitalCommons@USU. It has been accepted for inclusion in Conference Proceedings by an authorized administrator of DigitalCommons@USU. For more information, please contact digitalcommons@usu.edu.

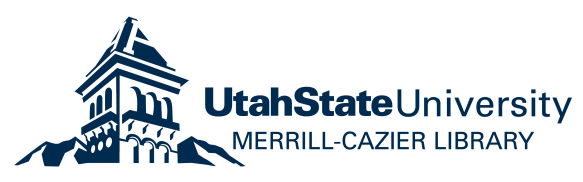




\title{
Defects Density of States Model of Cathodoluminescent Intensity and Spectra of Disordered $\mathrm{SiO}_{2}$
}

\author{
Amberly Evans Jensen and JR Dennison
}

\begin{abstract}
Electron beam measurements have been made that show disordered $\mathrm{SiO}_{2}$ exhibits luminescent behavior, and that it varies with incident beam energy and current density, sample temperature and wavelength. A simple model based on the electronic band structure and defect density of states-initially used to explain electron transport in highly disordered insulating materials - has been extended to predict the relative cathodoluminescent intensity and spectral radiance for disordered $\mathrm{SiO}_{2}$ as a function of these variables. Insulating $\mathrm{SiO}_{2}$ has a band gap of $\sim 8.9 \mathrm{eV}$. Hence thermal excitation from the valence to conduction band is highly improbable; excitation is through collisions of the incident high energy electrons. For visible and near-IR (NIR) light to be emitted, there must be other states within the forbidden band gap for electrons to occupy. These localized defect or "trap" states of disordered $\mathrm{SiO}_{2}$ are due to structural or substitutional chemical defects. Results for both thin film samples with penetrating radiation and thick bulk samples with nonpenetrating radiation were taken. The data were fit with the proposed model using saturation dose rate and mean shallow trap energy as fitting parameters, which can be compared with results from independent experiments.
\end{abstract}

Index Terms - cathodoluminescence, light emission, materials testing, space environment effects, low temperature

\section{INTRODUCTION}

$\mathrm{B}$ ecause spacecraft reside in the harsh space plasma environment, there is a need for studies on and understanding of material charging. Spacecraft charging is the accumulation of an electrical charge on orbiting spacecraft induced by the space plasma environment and has harmful effects on the electrical functionality of a spacecraft. It is one of the most destructive occurrences on spacecraft, causing more than half of all spacecraft problems due to the environment [1]. The space environment includes a dynamic mix of particle species, charged and neutral plasmas, electric and magnetic fields, radiation, and physical debris [2]. The effects of spacecraft interaction with this environment can

The studies were supported by funding from the NASA Goddard Space Flight Center. Additional support was provided by the Air Force Research Laboratory through a National Research Council Senior Research Fellowship for Dennison and a NASA Space Technology Research Fellowship for Jensen.

Amberly Evans Jensen (e-mail: Amb.Eva@aggiemail.usu.edu) and JR Dennison (e-mail: JR.Dennison@usu.edu) are with the Materials Physics Group in the Physics Department at Utah State University in Logan, UT 84322 USA. include a range of problems from damage to the craft's structure, degradation of the electronic components, to unwanted electrical behavior and, in extreme cases, loss of spacecraft [1]. In addition to charging induced by space plasma fluxes, incident electrons can also generate light emission. Such cathodoluminescence can potentially produce a stray light background in space-based astronomical observatories which are exposed to high energy electron fluxes from space plasmas. The magnitude of cathodoluminescence will be significantly affected by the environmental conditions, in particular the energy spectrum of impacting electrons and temperature. Energy spectra representative of four common space plasma environments (GEO, auroral, plasma sheet and solar wind), dominated by fluxes $\left(\sim 0.01 \mathrm{pA} / \mathrm{cm}^{2}\right.$ to $\left.\sim 10 \mathrm{nA} / \mathrm{cm}^{2}\right)$ for electron energies of up to $\sim 100 \mathrm{keV}$ [2-4]. Severe space weather conditions for such environments have on the order of $10 \mu \mathrm{W}-\mathrm{cm}^{-2}$ incident electron power (i.e., $10 \mathrm{nA}-\mathrm{cm}^{-2}$ and $1 \mathrm{keV}$ or $1 \mathrm{nA}-\mathrm{cm}^{-2}$ and $10 \mathrm{keV}$ ); this provides an absolute scale for comparison of luminescent intensities observed in the experiments described in these studies with space effects. In the case of IR observatories or sensors, the applications often require low temperatures down to 10's of Kelvin.

The objective of this study was to investigate cathodoluminescence of a common optical material, disordered silicon dioxide, under simulated space plasma conditions, using laboratory conditions in a space simulation chamber [5, 6]. Measurements were made of the absolute spectral radiance and optical emission spectra of this material, to quantify the effects of electron energy, current density and power density, temperature and sample thickness. Tests were conducted on two types of disordered $\mathrm{SiO}_{2}$ samples, a common optical coating, a thin optical coating on a reflective mirror with penetrating radiation and a thick bulk sample with nonpenetrating radiation. Absolute spectral radiance and emission spectra were measured over the full visible range and sample temperatures were varied from $\sim 295 \mathrm{~K}$ to $40 \mathrm{~K}$.

\section{EXPERIMENTATION}

These experiments were conducted in the main USU electron emission ultrahigh vacuum test chamber [7], modified for observations of low intensity ultraviolet/visable/near infrared (UV/VIS/NIR) glow over a broad range of sample temperatures $[6,8]$. A schematic of the experimental elements is shown in Fig. 2. 


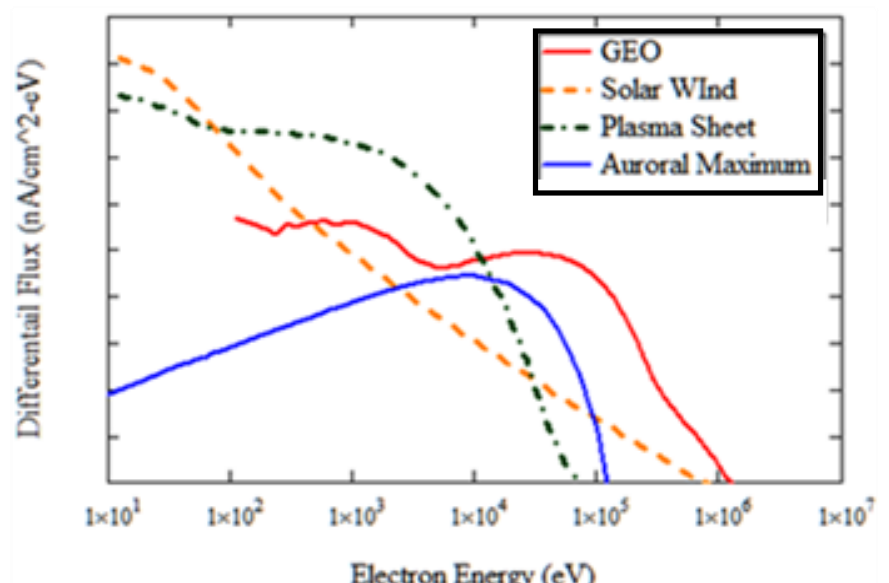

Figure 1. Typical space differential electron flux spectra for geostationary earth orbit [2], solar wind at the mean earth orbital distance [4], Earth plasma sheet [3] and a peak auroral storm [2].

Two monoenergetic electron sources were used. The low energy electron gun (Staib, model EK-5-51) produced a beam that ranges in energy of $20 \mathrm{eV}$ to $5000 \pm 2 \mathrm{eV}$ and flux of $\sim 50$ $\mathrm{pA}-\mathrm{cm}^{-2}$ to $1 \mu \mathrm{A}-\mathrm{cm}^{-2}$ [8]. The high energy gun (Kimball, model EGPS-21B) provided a flux of $\sim 50 \mathrm{pA}-\mathrm{cm}^{-2}$ to $1 \mu \mathrm{A}$ $\mathrm{cm}^{-2}$ over an energy range of 5.00 to $30.00 \pm 0.01 \mathrm{keV}$.

Light detection used two cameras, calibrated for NISTtracable absolute intensity measurements [6]. The cameras were positioned with clear views of the sample through vacuum port windows, thus allowing collection of photon emission data resulting from electron-induced luminescence and arcing [9]. The Single Lens Reflex (SLR) CCD camera (Cannon, EOS Rebel XT DS126071; 400 nm to $700 \mathrm{~nm}, 30$ frames per s) took visible light 10 Mpixel images at $30 \mathrm{~s}$ shutter speeds at full aperture with a $55 \mathrm{~mm}$ lens, giving it an average spectral response of $\sim 4 \cdot 10^{9}$ counts $/\left(\mathrm{W} / \mathrm{cm}^{2} \cdot \mathrm{sr} \cdot \mu \mathrm{m}\right)$. The image-intensified CCD video camera (Xybion, ISG-780U-3; $400 \mathrm{~nm}$ to $900 \mathrm{~nm}$ ) collected data at 30 frames per sec using a $55 \mathrm{~mm}$ lens with a spectral response of $\sim 4 \cdot 10^{10}$ counts $/\left(\mathrm{W} / \mathrm{cm}^{2} \cdot \mathrm{sr} \cdot \mu \mathrm{m}\right)$. A fiber optics-based spectrometer (Stellarnet, 13LK-C-SR; $250 \mathrm{~nm}$ to $1080 \mathrm{~nm}$ ) provided UV/Vis spectral measurements with a wavelength resolution of $\sim 1 \mathrm{~nm}$. Because the luminescence is such low intensity light, the spectra were acquired at its longest integration time, $\sim 65 \mathrm{~s}$, to collect spectra.

Two options for sample cooling were used. In the first, the sample carousel was thermally anchored to (but electrically isolated from) a liquid $\mathrm{N}_{2}$ cryogen reservoir equipped resistive heaters; the samples were maintained over a range of temperatures from $\sim 150 \mathrm{~K}$ to $\sim 400 \mathrm{~K}$ with a stability of $\pm 4 \mathrm{~K}$ maintained over typical $2 \mathrm{hr}$ experiment durations [5]. The other cooling option used a closed-cycle helium cryostat with operational sample temperatures from $<40 \mathrm{~K}$ to $>450 \mathrm{~K}$. Temperature is maintained to $\pm 0.5 \mathrm{~K}$ using a standard computer-controlled PID temperature controller under Labview ${ }^{\mathrm{TM}}$ command and platinum resistors [6].

Two types of disordered $\mathrm{SiO}_{2}$ samples were tested. The first sample type had a thin film ( $\sim 60 \mathrm{~nm}$ thick) coating deposited on a gold-coated, optically flat mirror surface using plasma vapor depositon. The second sample type was a bulk sample, $\sim 80 \mu \mathrm{m}$ thick, made by traditional glass formation methods. The samples were optically cleaned and underwent a $\sim 12 \mathrm{hr}$

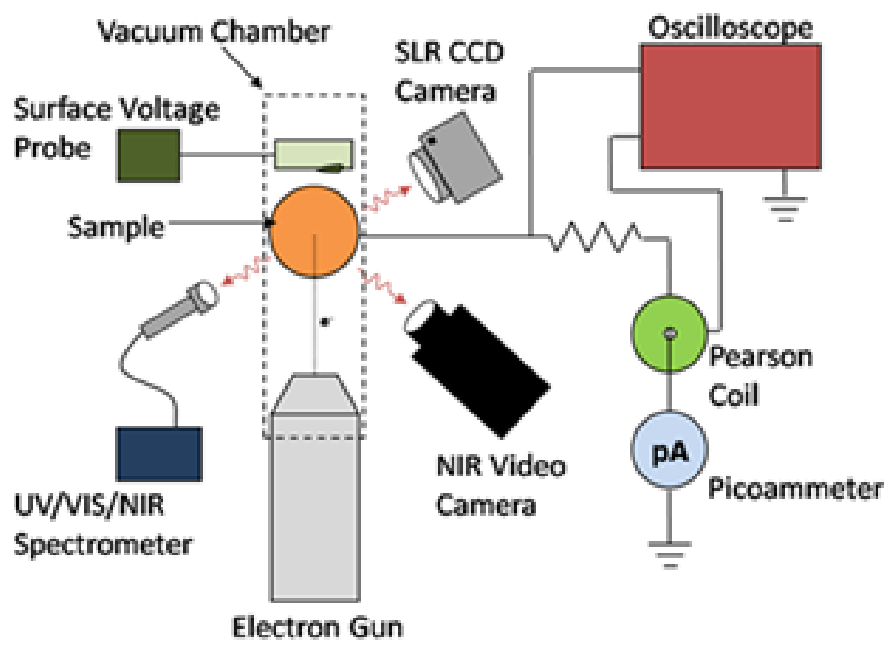

Figure 2. Block diagram of instrumentation for collecting cathodeluminescence and spectral data induced by electron beam bombardment, electrode current, and the surface voltage. Instrumentation included an SLR Vis CCD still camera, a Vis/NIR CCD video camera and UV/Vis and IR spectrometers for optical measurements plus picoammeters, Pearson coils, and a storage oscilloscope for electrode current measurements [6, 8].

vacuum bakeout at $\sim 390 \mathrm{~K}$ and $<1 \cdot 10^{-3} \mathrm{~Pa}$ to eliminate adsorbed water and volatile contaminates. The samples were mounted and placed in an ultrahigh vacuum chamber (base pressure $<1 \cdot 10^{-6} \mathrm{~Pa}$ ) for $>24 \mathrm{hr}$ to allow for outgassing before measurements were made.

\section{CATHOdoluminescence Model}

A model for the cathodoluminescence for highly disordered insulating materials has been developed by Jensen and Dennison, based on band models of the localized disordered trap states found in the forbidden bandgap region of insulating materials $[9,10]$. It is an extension of theories for both band structures of defects states in crystalline materials and luminescence in atomic and crystalline materials [11]. Incident high energy electrons excite electrons from the valence band into the conduction band, which quickly are trapped into shallow trap states just below the conduction band edge at $E_{c}$. These electrons in shallow trap states can decay into deep level traps and emit luminescence photons. The luminescent intensity $I_{\gamma}$ is proportional to the number of available states and the transition rates of each phase depicted in the modified Joblonski diagram [11,12] of Figure 3.

The model predicts that $I_{\gamma}$ scales with incident current density, $J_{b}$, incident beam energy $E_{b}$, temperature $T$, and photon wavelength $\lambda$ as

$$
\begin{gathered}
I_{\gamma}\left(J_{b}, E_{b}, T, \lambda\right) \propto \frac{\dot{D}\left(J_{b}, E_{b}\right)}{\dot{D}+}\left\{\left[e^{-\left(\varepsilon_{S T} / k_{B} T\right)}\right]\left[-e^{-\left(\varepsilon_{S T} / k_{B} T\right)}\right]\right\} \\
\times\left\{\left[1-\mathbb{A}_{f}(\lambda)\right]\left[1+\mathbb{R}_{m}(\lambda)\right]\right\}
\end{gathered}
$$

$\dot{D}$ is the dose rate, or absorbed power per unit mass. The dose rate is given by

$$
\dot{D}\left(J_{b}, E_{b}\right)=\frac{E_{b} J_{b}\left[1-\eta\left(E_{b}\right)\right]}{q_{e} \rho_{m}} \times\left\{\begin{array}{cc}
{[1 / L]} & ; R\left(E_{b}\right)<L \\
{\left[1 / R\left(E_{b}\right)\right]} & ; R\left(E_{b}\right)>L
\end{array}\right.
$$




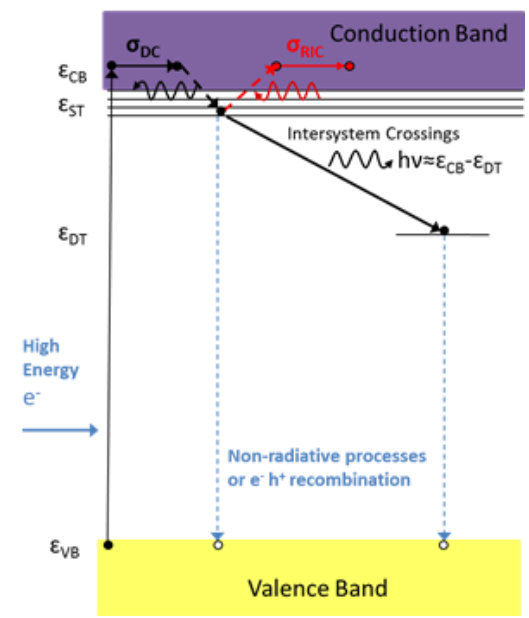

Figure 3. Modified Joblonski diagram for electron-induced phosphorescence. Shown are transitions between the extended state valence (VB) and conduction (CB) bands, shallow trap (ST) states at $\varepsilon_{\mathrm{ST}}$ within $\sim k_{B} T$ below the $\mathrm{CB}$ edge, and deep trap (DT) distributions centered at $\varepsilon_{\mathrm{DT}}$. Energy depths are exaggerated for clarity.

where $q_{e}$ is the electron charge, $\rho_{m}$ is the mass density of the material, and $L$ is the sample thickness. At high dose rates, saturation is observed, characterized by a material and sample dependent saturation dose rate, $\dot{D}_{\text {sat }}$.

$R\left(E_{b}\right)$ is the energy-dependent penetration depth or range, which is a material-dependent property. Fig. 4 shows the range of $\mathrm{SiO}_{2}$ as a function of incident electron energy calculated in the continuous slow down approximation [13], and the associated dose rate for $10 \mathrm{nA}-\mathrm{cm}^{-2}$ incident current density. When the range is less than the sample thickness, the beam is penetrating and all the incident power is absorbed in the material. Penetrating radiation occurs when the range is greater than the sample thickness and the absorbed power is reduced by a factor of $\left[L / R\left(E_{b}\right)\right][10]$.

Inclusion of a weakly energy-dependent backscatter coefficient, $\eta\left(E_{b}\right)$, in Eq. (2) corrects the dose rate for incident electrons that quasi-elastically backscatter without depositing appreciable energy in the material [10]. For the most part, this correction is small and weakly dependant on energy. For biased samples, or when excess charge is stored in the trap states, a surface voltage $V_{s}$ results and $E_{b}$ is replaced everywhere in Eqs. (1) and (2) by the landing energy, $\left(E_{b}-\right.$ $\left.q_{e} V_{s}\right)$. Thus, glow intensity may diminish appreciably for $\mathrm{kV}$ surface potentials often observed on GEO satellites in eclipse. In the calculations presented here, this affect was assumed to be negligible.

The thermal dependence of luminescence is characterized by the mean energy depth of the shallow traps below the conduction band, $\varepsilon_{S T}$. In equilibrium, it is proportional to the number of electrons in the conduction band that can fall into the shallow traps $\left(\propto\left[e^{-\left(\varepsilon_{S T} / k_{B} T\right)}\right]\right)$ and to the fraction of electrons that are retained in the shallow traps and not thermally excited into the conduction band $(\propto[1-$ $e-\varepsilon S T k B T)$. Thus, I $\gamma \propto e-\varepsilon S T k B T 1-e-\varepsilon S T k B T$. At higher temperatures where $k_{B} T \gg \varepsilon_{S T}$, the thermal energy exceeds $\varepsilon_{S T}$, and $I_{\gamma} \propto\left(\varepsilon_{S T_{Q}} / k_{B} T\right)$.

The final term in curly brackets in Eq. (1) accounts approximately for the wavelength, $\lambda$, dependent photons propagation through the coating. The optical absorption of the

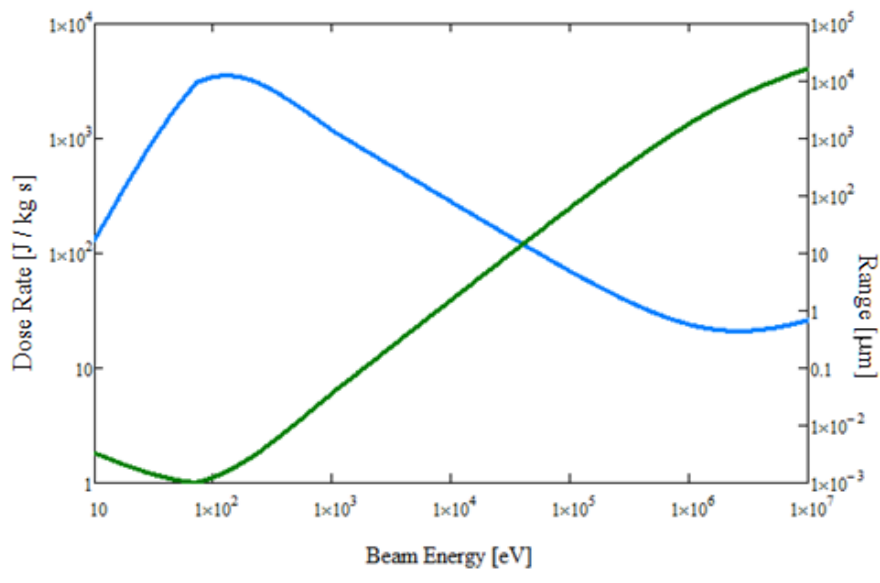

Figure 4. Range (green) and dose rate (blue) of disordered $\mathrm{SiO}_{2}$ as a function of incident energy using the continuous slow-down approximation, based on calculations from [13].

$\mathrm{SiO}_{2}$ coating materials is $\mathbb{A}_{f}(\lambda) \ll 1$ for propagation through the full film; thus it does not attribute significantly for these measurements. $\mathbb{R}_{m}(\lambda)$ is the reflectivity of the underlying metallic layer. For highly reflective materials like Ag, $\mathbb{R}_{m} \approx 1$ at all $\lambda$ considered. For materials like $A u$, the glow spectrum is enhanced at longer $\lambda$, due to greater absorptivity of $\mathrm{Au}$ at shorter $\lambda$.

\section{RESULTS}

The measured dependence of spectral radiance on $J_{b}, E_{b}, T$, and $\lambda$ and the emission spectra are described below. Fits to the data determine values of $\varepsilon_{S T}$, and $\dot{D}_{\text {sat }}$ and details about the band structure of disordered $\mathrm{SiO}_{2}$.

\section{A. Incident Beam Current Density}

For $\mathrm{SiO}_{2}$ (band gap $\approx 8.9 \mathrm{eV}$ ), excitation from the valence band to conduction band is not thermal, but rather occurs through collisions with the incident high energy electrons. Thus, at low currents, $I_{\gamma}$ is proportional to the incident current, $J_{b}$, through the dose rate. At very high current density, saturation can occur when trap states fill, limiting the number of states electrons can decay into, and leading to $I_{\gamma}$ approaching a constant value.

Current dependent saturation is shown in Fig. 5, where the spectral radiance as a function of beam current density for various incident beam energies is fit using Eqs. (1) and (2). The departures from the initial linear dependence on current density is attributable to saturation effects at higher current density when trap states fill, limiting the number of states electrons can decay into. This is most pronounced in Fig. 5(a) for the thin sample with a $\dot{\mathrm{D}}_{\text {sat }}$ value of $4.4 \cdot 10^{3} \pm 44 \% \mathrm{~Gy} / \mathrm{s}$. Saturation effects are present, though much reduced, for the thick sample $\dot{\mathrm{D}}_{\text {sat }}$ is $577 \pm 25 \% \mathrm{~Gy} / \mathrm{s}$. The smaller saturation dose rate for the thicker sample may well be a result of the preparation method for the samples; the vapor deposited thin sample would be expected to have substantially more defects than the thick sample and more defects would require a higher dose rate to produce saturation. Note that as the dose rate changes from thickness dependent (thick sample) to range dependent (thin sample), $\dot{\mathrm{D}}_{\text {sat }}$ increases dramatically. 

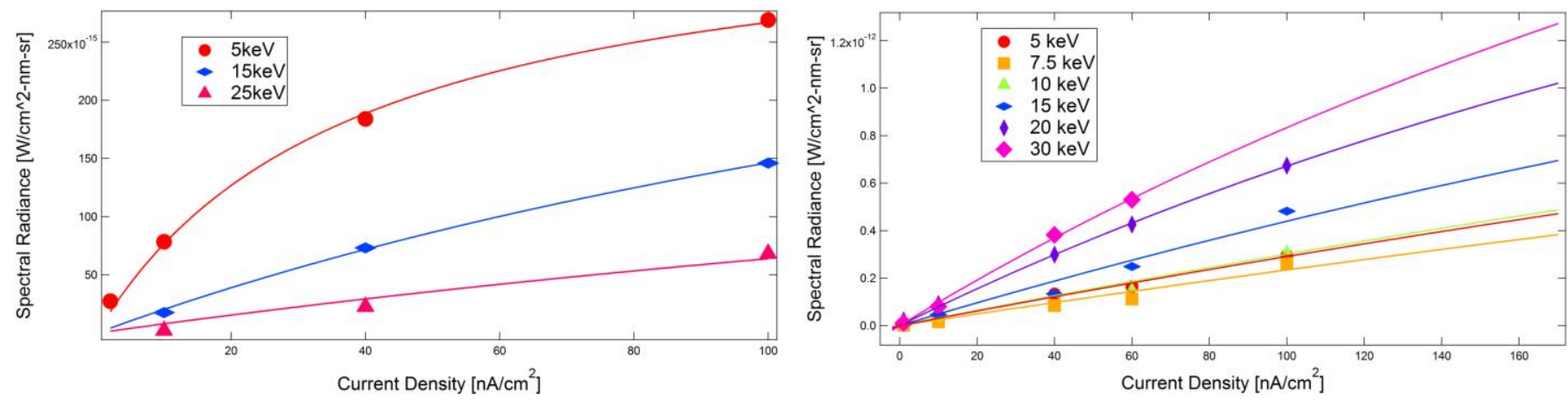

Figure 5. Spectral radiance as a function of beam current density for various incident electron energies. (a) For the $\sim 60 \mathrm{~nm}$ thick sample of $\mathrm{SiO}_{2}$, penetration occurs at $\sim 1.3 \mathrm{keV}$ beam energy. The fits yield a value for $\dot{\mathrm{D}}_{\text {sat }}$ of $4.4 \cdot 10^{3} \pm 44 \% \mathrm{~Gy} / \mathrm{s}$. (b) For the $\sim 80 \mu \mathrm{m}$ thick sample of $\mathrm{SiO}_{2}$, penetration occurs at $\sim 118 \mathrm{keV}$ beam energy. The fits yield a value for $\dot{\mathrm{D}}_{\text {sat }}$ of $577 \pm 25 \% \mathrm{~Gy} / \mathrm{s}$.

\section{B. Incident Beam Energy}

Below saturation current densities, $I_{\gamma}$ is linearly proportional to $\dot{D}$, as given by Eq. (2). For nonpenetrating radiation, the unsaturated intensity $I_{\gamma}$ and $\dot{D}$ are linearly proportional to the incident power density, $\left(J_{i n c} E_{b} / q_{e}\right)$, and to $E_{b}$. This behavior is depicted by the green curves in Fig. 6 . For penetrating radiation, the unsaturated intensity $I_{\gamma}$ scales as $\left[E_{b} / R\left(E_{b}\right)\right]$, as shown by the blue curves in Fig. 6. The intersection of these two curves occurs at the penetration beam energy at which the range, $R\left(E_{b}\right)$, equals the thickness of the sample, $L$. Thus, the expected luminescent behavior follows the solid portions of each curve and the dashed curves predict the penetrating and nonpenetrating behavior outside their energy ranges of applicability. The penetration energy, based on the range curve in Fig. 4, is denoted by the vertical purple curve $(\sim 1.3 \mathrm{keV}$ for the $\sim 60 \mathrm{~nm}$ sample and $\sim 118 \mathrm{keV}$ for the $\sim 80 \mu \mathrm{m}$ sample). For the thick sample, shown in Fig. 6(b), the maximum beam energy used in the experiments was $30 \mathrm{keV}$, so the experiments were all nonpenetrating. Measured data (circles) at a constant $10 \mathrm{nA}-\mathrm{cm}^{-2}$ shown in Fig. 6 follow the nonpenetrating portion of the predicted curve for the thick sample and the penetrating portion for the thin sample. The fits in Fig. 6 were obtained using the $\dot{\mathrm{D}}_{\text {sat }}$ value calculated from the previous section related to incident beam current

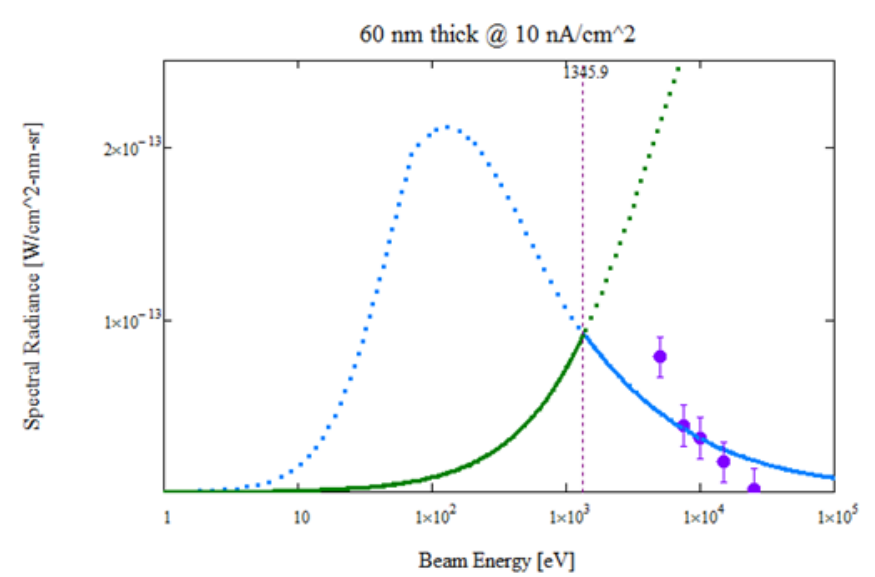

density.

The order of the amplitudes of the curves in Fig. 5, increasing amplitudes with increasing energy for the nonpenetrating thick sample in Fig. 5(a) and decreasing amplitudes with increasing energy for the penetrating curves in Fig. 5(b), reflect the energy-dependant behavior described above.

\section{Temperature}

Fig. 8 shows the temperature dependence of the $\mathrm{SiO}_{2}$ spectral radiance for the two samples measured at $5 \mathrm{keV}$ and $>200 \mathrm{nA}-\mathrm{cm}^{-2}$, fit with Eq. (1). As predicted, the spectral radiance of the thin sample in Fig. 8(a) increases exponentially as the temperature decreases until it reaches a maximum value, and then begins to decrease. The peak temperature is $67 \mathrm{~K}$ with a $\varepsilon_{S T}=4 \mathrm{meV}$. The data for the thick sample in Fig. 8(b) do not exhibit decreasing intensity at lower $\mathrm{T}$, but is still fit well with Eq. (1) with $\varepsilon_{\mathrm{ST}}=1 \mathrm{meV}$, which predicts a peak temperature of $16 \mathrm{~K}$. The observed differences in temperature dependence of the two samples is consistent with the idea that the thicker sample with lower defect density would have a higher effective temperature, as trapped electrons collect in the shallow trap distribution.

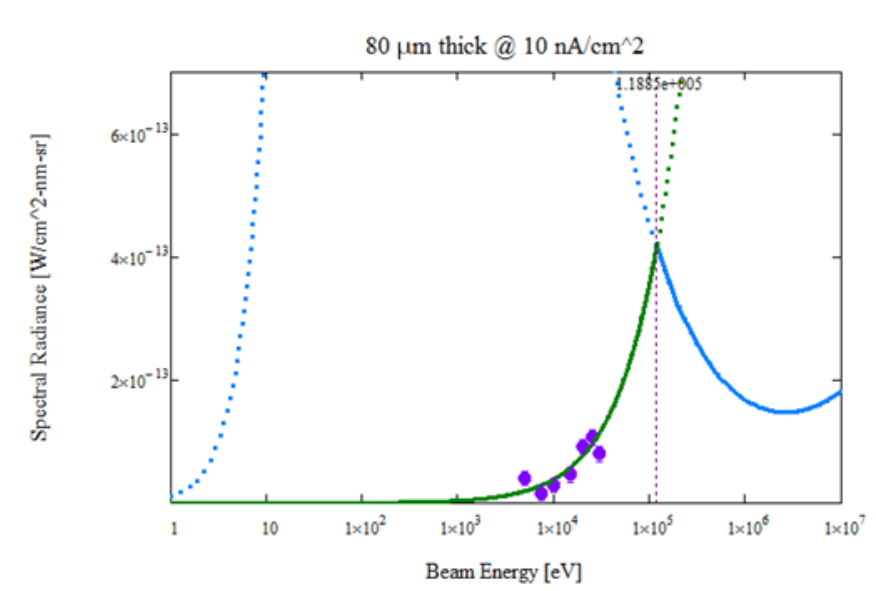

Figure 6. Spectral radiance relationship with beam energy at $10 \mathrm{nA} / \mathrm{cm}^{2}$. The green curve is the behavior of the spectral radiance when the energy dependent range is less than the material thickness; all power is deposited into the sample. The blue curve is the behavior of the spectral radiance when the energy dependent range is greater than the material thickness. The vertical purple dotted line is the energy at which the range is equal to the thickness of the sample, the penetration energy. These curves were created using the range function (Figure 4) and Eqns 1 and 2. The luminescent behavior follows the solid portions of the curves, not the dotted portions. (a) For the $\sim 60 \mathrm{~nm}$ sample, the penetration energy is $\sim 1.3 \mathrm{keV}$ and the range is greater than the sample thickness, decreasing with increasing energy. (b) For the $\sim 80 \mu \mathrm{m}$ sample, the penetration energy is $\sim 118 \mathrm{keV}$ and the range was less than the sample thickness, increasing with energy. 

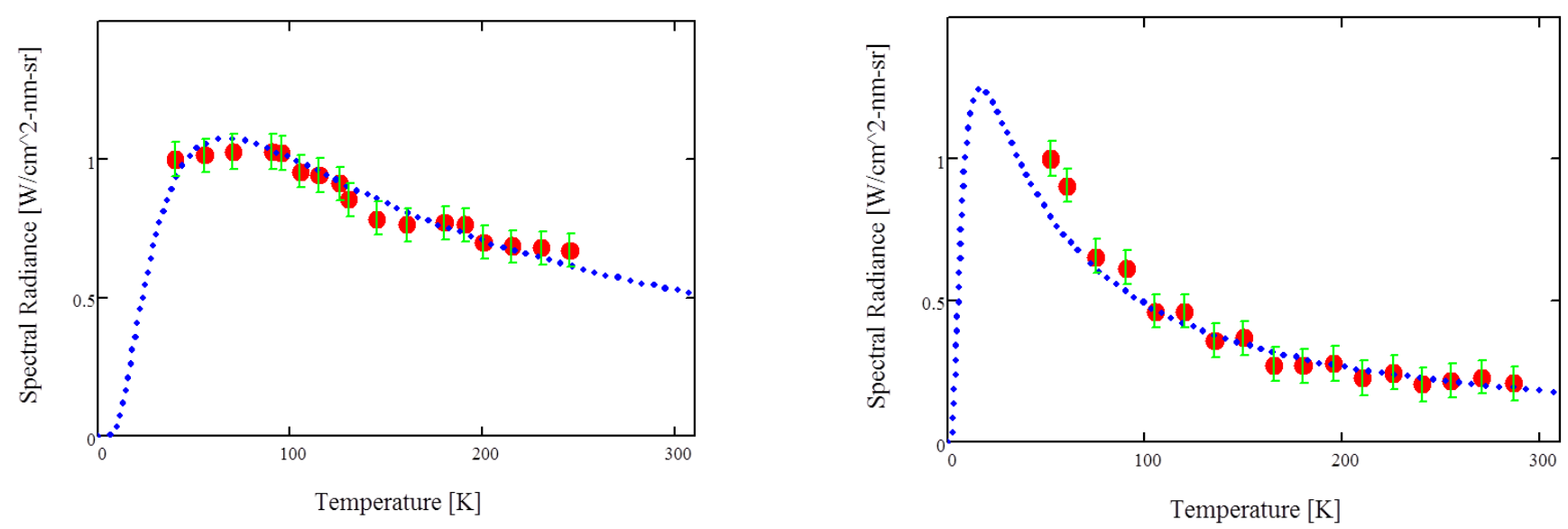

Figure 7. The spectral radiance of $\mathrm{SiO}_{2}$ as a function of sample temperature, measured at $5 \mathrm{keV}$ and $>200 \mathrm{nA}-\mathrm{cm}^{-2}$. Curves are fit with Eq.(1). (a) $60 \mathrm{~nm}$ sample, fit with a $\varepsilon_{S T}=4 \mathrm{meV}$, with a maximum temperature of $67 \mathrm{~K}$. (b) $80 \mu \mathrm{m}$ sample, fit with $\varepsilon_{S T}=1 \mathrm{meV}$, with a peak temperature of $16 \mathrm{~K}$.

\section{Wavelength}

Temperature dependent emission spectra in the visible range were also collected for thin and thick samples. The raw spectral data were fit with three Gaussian functions to give the spectra shown in Fig. 8. The spectra for the thin sample (Fig. $8(\mathrm{a})$ ) and the thick sample (Fig. 8(b)) both had two dominant bands centered at approximately $500 \mathrm{~nm}$ and $645 \mathrm{~nm}$; an additional shoulder was observed at $\sim 455 \mathrm{~nm}$ at low temperature (Fig. 8). A fourth peak in the UV range at $\sim 275$ $\mathrm{nm}$ was observed for the thin samples [9]; the UV range was not measured for the thick sample. The positions of these observed peaks are consistent with the results of Sahl [14] and Trukhin [15] for similar disordered $\mathrm{SiO}_{2}$ samples at room temperature. Sahl identified the peak at $645 \mathrm{~nm}$ as a nonbridging oxygen hole center in the structure. The peak at $500 \mathrm{~nm}$ and the $275 \mathrm{~nm}$ peak are a result of an oxygen deficient center, as identified by Trukhin. The origin of an additional shoulder at $\sim 455 \mathrm{~nm}$ has not yet been identified. As temperature changes, the peak positions remain relatively constant, except where the $455 \mathrm{~nm}$ and $500 \mathrm{~nm}$ peaks become indistinguishable.

The peak widths remain relatively constant with changing temperature but vary from one peak to another. Note the peak widths of the two peaks attributed to the oxygen deficient centers (Fig.9) have much larger widths than the other two peaks [9], which may reflect broader distributions for this type of defect. Alternately, the broader peaks may actually be made up of several overlapping bands.

Fig. 9 shows a simplified qualitative representation of the

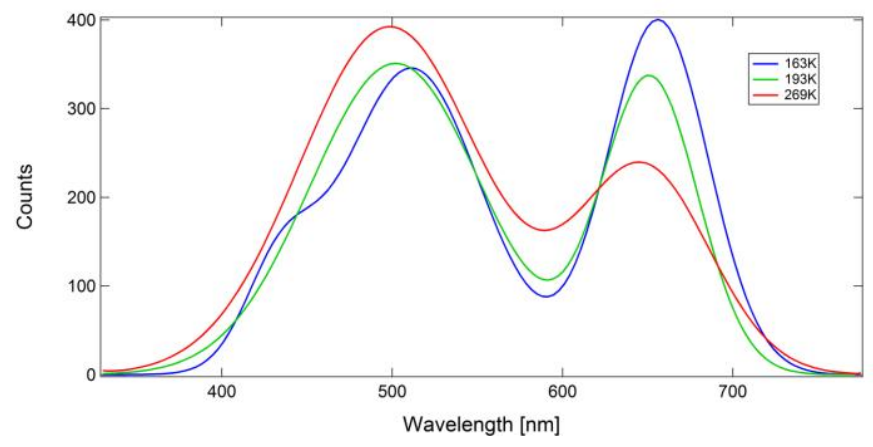

$\mathrm{SiO}_{2}$ density of states for deep level traps. The bands depicted are Gaussian in shape, with depths, widths and intensities based approximately on observed spectral peaks for bulk $\mathrm{SiO}_{2}$ at $\sim 55 \mathrm{~K}$. The four peaks in the disordered $\mathrm{SiO}_{2}$ luminescence spectra are attributed to bands of localized defect or "deep trap" (DT) states, at $\sim 1.93, \sim 2.48, \sim 2.76$, and $\sim 4.97 \mathrm{eV}$ below the conduction band edge [9].

Fig. 10 displays the normalized peak intensities for the 500 $\mathrm{nm}$ (green) and $645 \mathrm{~nm}$ (red) peaks as a function of temperature, for both thin and thick samples. As temperature decreased, the intensity of the red band continually increased. The green band intensity originally decreased as the temperature decreased, but began to increase below $\sim 165 \mathrm{~K}$. This same peak behavior was also seen in the still camera images, where the red, green and blue components of the color images followed the same temperature dependent trends as the corresponding peaks in the spectra [9].

These behaviors are attributed to the thermal filling of the deep traps [10]. (For disordered materials, the peak amplitudes are roughly proportional to the density of states for the associated defect since there are no symmetry selection rules for disordered states. Further, the spectrometer response function is relatively flat over the spectral range shown in Fig. 10.) The temperature dependence for a given distribution may depend on the position of the effective Fermi level, which increases at higher temperatures, as described in [16]. At low temperature the higher energy defect bands will be largely unoccupied, while those bands below the effective Fermi level will be mostly filled. As $\mathrm{T}$ increases, thermal energy excites electrons in lower bands into higher states, thus enhancing the

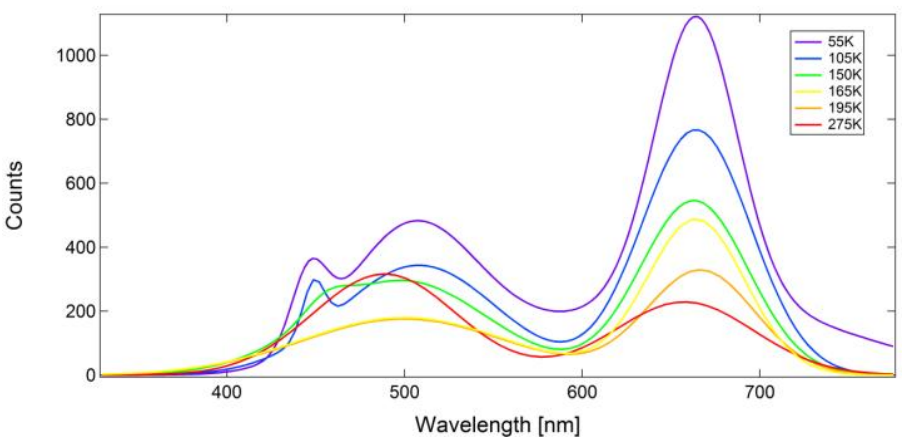

Figure 8. Fits to measured emission spectra were obtained at various sample temperature. The spectra have two peaks centered at $\sim 500 \mathrm{~nm}$ and $\sim 645 \mathrm{~nm}$; an additional shoulder is observed at $\sim 455 \mathrm{~nm}$. (a) Spectra are from the $\sim 60 \mathrm{~nm}$ sample, cooled to $\sim 160 \mathrm{~K}$. (b) Spectra from the $\sim 80 \mu \mathrm{m}$ sample, cooled to $\sim 55 \mathrm{~K}$. 


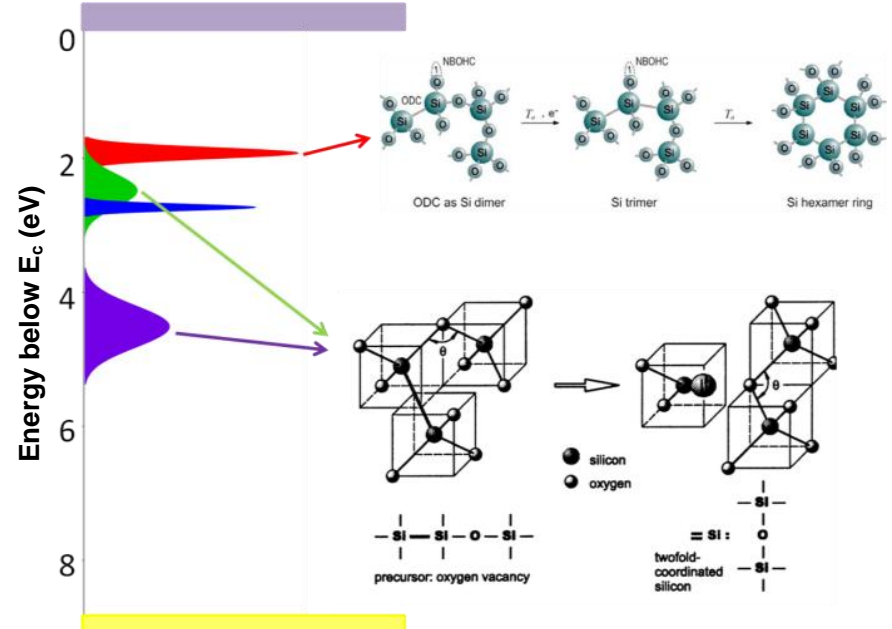

Figure 9. Simplified qualitative representation of the $\mathrm{SiO}_{2}$ density of states for deep level traps. The bands depicted are Gaussian in shape, with widths and intensities based approximately on observed spectral peaks for bulk $\mathrm{SiO}_{2}$ at $\sim 55 \mathrm{~K}$. At least four peaks in the disordered $\mathrm{SiO}_{2}$ luminescence spectra (see Fig. 8) are attributed to bands of localized defect or "deep trap" (DT) states, at $\sim 1.93, \sim 2.48, \sim 2.76$, and $\sim 4.97 \mathrm{eV}$ below the conduction band edge [9]. $\mathrm{SiO}_{2}$ studies at room temperature have attributed a $645 \mathrm{~nm}$ (red) peak to a nonbridging oxygen hole center [14] and $275 \mathrm{~nm}$ (purple) and $500 \mathrm{~nm}$ (green) peaks to an oxygen deficient center [15]. The origin of an additional (blue) shoulder at $\sim 455 \mathrm{~nm}$ has not yet been identified.

probability for relaxation of conduction band and shallow trapped electrons into the lower bands and the emission of higher energy photons. This can explain the decrease in the red peak intensity at higher temperatures. The more complicated behavior of the green peak may result from the effective Fermi level moving through the band associated with the green peak; it may also be complicated by the close vicinity to the much narrower blue peak, which shows up as a small shoulder off the green peak at low temperatures.

\section{CONCLUSIONS}

Low intensity cathodoluminescence was observed for disordered $\mathrm{SiO}_{2}$ thin film and bulk samples under low intensity incident electron irradiation. A model based on multilevel disordered peak DOS explains the intensity versus beam current density, beam energy and material temperature trends. As the current density increased, the luminescent intensity increased until it reached a saturation level. Using the model, this saturation level was determined and then used in the energy dependent analysis. When the range was less than the sample thickness, at sufficiently low energy beam, the luminescent intensity increased linearly with energy. However, once the range exceeded the sample thickness, the intensity fell off with increasing energy. The model also extends to the temperature dependent behavior of cathodoluminescence. After initially increasing with decreasing temperature, the overall intensity falls off exponentially with increasing temperature. The observed number and wavelengths of the peaks were consistent with previous observations. The intensity and peak positions of the primary peaks observed in the visible region were measured as a function of temperature from $\sim 55 \mathrm{~K}$ to $\sim 280 \mathrm{~K}$. Using these fits from the model, the material and sample properties

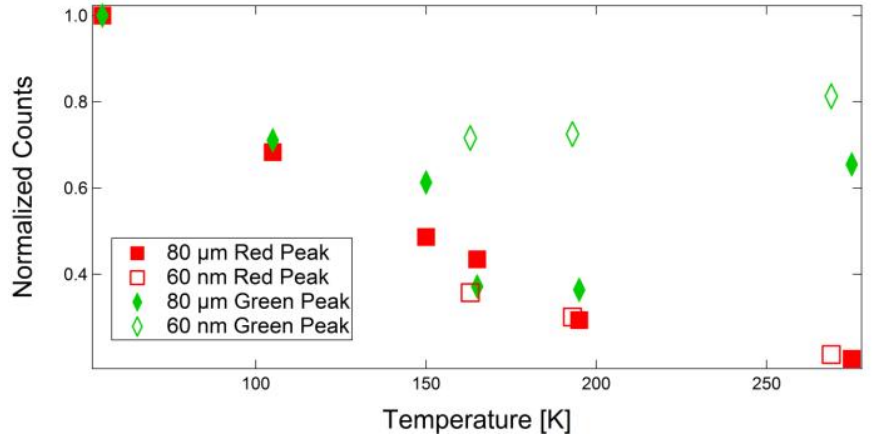

Figure 10. Temperature dependent behavior of the red and green spectral peaks, normalized to the most intense peak value. The red $650 \mathrm{~nm}$ peak continually decrease with increasing temperature. The green $500 \mathrm{~nm}$ peak also begins decreasing with increasing temperature but starts to increase as temperature approaches $165 \mathrm{~K}$. Separate data are shown for the thin (open symbols) and thick (solid symbols) data.

relating to the defect density of states, $\dot{D}_{s a t}$ and $\varepsilon_{\mathrm{ST}}$, were determined.

The results of these experiments suggest that materials used in structural components, optical elements, and thermal control surfaces of spacecraft and space-based observatories could, when exposed to sufficiently energetic, charged particles from the space plasma environment, luminesce [1719]. If these visible, infrared and ultraviolet emissions are intense enough, they could potentially produce optical contamination detrimental to the performance of the observatory's optical elements and sensors, and act to limit their sensitivity and performance windows. The use of this model can be and is being extended to other types of materials [20]. By extending it to other materials, this model becomes an integral component in understanding the affect of the space environment on many types of spacecraft.

\section{ACKNOWLEDGEMENT}

We gratefully acknowledge contributions to instrumentation from Tamara Jeppsen, Michael Taylor for the use of infrared and CCD video cameras, and useful discussions with Robert Meloy and Charles Bowers of NASA Goddard Space Flight Center. We are also grateful for the help, insight and contribution of every member of the Materials Physics Group, especially that of Justin Dekany and Greg Wilson for their hours of experimental set-up, data collection and analysis.

\section{REFERENCES}

[1] R. Leach and M. Alexander, "Failures and anomalies attributed to spacecraft charging," NASA STI/Recon Technical Report N, vol. 96, p. $11547,1995$.

[2] D. Hastings and H. Garrett, Spacecraft-Envrionment Interactions. New York, NY: Cambridge Press, 1996.

[3] J. Minow, W. C. Blackwell Jr., and A. Dickmann, "Plasma Environment and Models for L2," AIAA $42^{\text {nd }}$ Aerospace Sciences Meeting and Exhibit, 2004.

[4] J. Minow, L. N. Parker, R. L. Alstaff, W. C. Blackwell Jr., and A. Dickmann, "Radiation and internal charging environments for thin dielectrics in interplanetary space," in $9^{\text {th }}$ Spacecraft Charging Technology Conference, Epochal Tsukuba, Tsukuba, Japan, 2005.

[5] W. Y. Chang, J. R. Dennison, N. Nickles, and R. E. Davies, "Utah State University Ground-based Test Facility for Study of Electronic Properties of Spacecraft Materials," in 6th Spacecraft Charging Technology Conference Air Force Research Laboratory Science Center, Hanscom Air Force Base, MA, 1998. 
[6] J. Dekany, R. H. Johnson, G. Wilson, A. Evans, and J. R. Dennison, "Ultrahigh Vacuum Cryosat System for Extended Low Temperature Space Environment Testing," IEEE Trans. on Plasma Sci, vol. 42, pp. 266-271, 2014.

[7] W. Y. Chang, J. Dennison, N. Nickles, and R. E. Davies, "Utah State University Ground-based Test Facility for Study of Electronic Properties of Spacecraft Materials," in 6th Spacecraft Charging and Technology Conference, Sir Force Research Laboratory Science Center, Hanscom Air Force Base, MA, 2000.

[8] G. Wilson, "The Internal Charge Evolution of Multilayered Dielectrics Undergoing Mono-Energetic Electron Bombardment," in Physics Department. vol. Masters Logan, UT: Utah State University, 2013.

[9] A. E. Jensen, G. Wilson, J. Dekany, A. Sim, and J. R. Dennison, "Low Temperature Cathodoluminescence of Space Observatory Materials," IEEE Trans. on Plasma Sci, vol. 42, pp. 305-310, 2014.

[10] J. R. Dennison, A. Evans, G. Wilson, C. W. Bowers, and R. Meloy, "Electron Beam Induced Luminescence of $\mathrm{SiO}_{2}$ Optical Coatings," in Conference on Electrical Insulation and Dielectric Phenomena Montreal, Canada, 2012.

[11] Lakowicz, "Principles of Flourescnece Spectroscopy," Plenum Press, 1983.

[12] A. Joblonski, "Uber den Mechanismmus des photoluminescence voFarbsoff-phosphoren," Z. Phys., vol. 94, pp. 33-46, 1935.

[13] G. Wilson and J. R. Dennison, "Approximation of Range in Materials as a Function of Incident Electron Energy," Plasma Science, IEEE Transactions on, vol. 40, pp. 291-297, 2012.

[14] R. Salh, A. v. Czarnowski, M. V. Zamoryanskaya, E. V. Kolesnikova, and $\mathrm{H}$. J. Fitting, "Cathodoluminescence of $\mathrm{SiO}_{\mathrm{x}}$ under-stoichiometric silica layers," Physica Status Solidi (a), vol. 203, pp. 2049-2057, 2006.

[15] A. N. Trukhin, M. Goldberg, J. Jansons, H. J. Fitting, and I. A. Tale, "Silicon dioxide thin film luminescence in comparison with bulk silica," Journal of Non-Crystalline Solids, vol. 223, pp. 114-122, 1998.

[16] J. R. Dennison, J. Dekany, J. C. Gillespie, P. Lundgreen, A. Anderson, A. E. Jensen, G. Wilson, A. Sim, and R. Hoffman, "Synergistic models of electron emission and transport measurements of disordered $\mathrm{SiO}_{2}$," in 13th Spacecraft Charging Technology Conference, Pasadena, CA, 2014.

[17] V. Griseri, L. A. Dissado, J. C. Fothergill, C. Laurent, and G. Teyssedre, "Photoluminescence, recombination induced luminescence and electroluminescence in epoxy resin," Journal of Physics D: Applied Physics, vol. 34, 2001.

[18] H. J. Fitting, T. Barfels, A. N. Trukhin, and B. Schmidt, "Cathodoluminescence of crystalline and amorphous $\mathrm{SiO}_{2}$ and $\mathrm{GeO}_{2}$," Journal of Non-Crystalline Solids, vol. 279, pp. 51-59, 2001.

[19] A. Evans, G. Wilson, and J. Dennison, "Temperature Dependence of $\mathrm{SiO}_{2}$ Electron-Induced Luminescence," in American Physical Society Four Corners Section Meeeting University of Arizona, Tucson, AZ: American Physical Society Four Corners Section Meeeting, 2011.

[20] J. Dekany, J. Christensen, J. R. Dennison, A. E. Jensen, G. Wilson, T. Schneider, C. W. Bowers, and R. H. Meloy, "Variations in cathodoluminescent intensity of spacecraft materials exposed to energetic electron bombardment," in 13th Spacecraft Charging Technology Conference, Pasadena, CA, 2014.

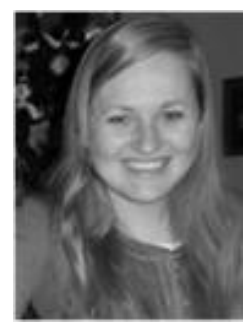

Amberly Evans Jensen is currently a graduate student at Utah State University in Logan, UT pursing an MS in physics. She received BS degrees in physics and chemistry from USU in 2012. She has worked with the Materials Physics Group for seven years on electron emission, luminescence and resistivity studies and on MISSE retrieval and post-flight analysis of SUSpECS. Much of her work has focused on optical scattering and emission of spacecraft materials.

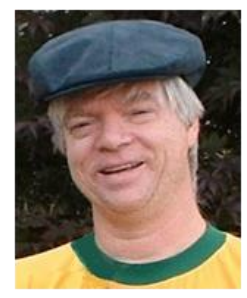

J. R. Dennison received the B.S. degree in physics from Appalachian State University, Boone, NC, in 1980, and the M.S. and Ph.D. degrees in physics from Virginia Tech, Blacksburg, in 1983 and 1985, respectively. He was a Research Associate with the University of Missouri-Columbia before moving to Utah State University (USU), Logan, in 1988. He is currently a Professor of physics at USU, where he leads the Materials Physics Group. He has worked in the area of electron scattering for his entire career and has focused on the electron emission and conductivity of materials related to spacecraft charging for the last two decades. 